\title{
Modus operandi Del Nuevo Consenso Macroeconómico en Brasil, Chile y MÉxico
}

\author{
Aída García Lázaro e Ignacio Perrotini*
}

Fecha de recepción: 01 de abril de 2014. Fecha de aceptación: 24 de junio de 2014.

\begin{abstract}
RESUMEN
Se analiza críticamente la experiencia de Brasil, Chile y México con la política monetaria de objetivos de inflación del Nuevo Consenso Macroeconómico (NCM). Mediante modelos de cointegración y corrección de errores y la discusión de los principales hechos estilizados contrastamos el NCM con la evidencia empírica disponible. Una característica esencial de su modus operandi en estas economías estriba en que: el control de la inflación depende crucialmente de la apreciación del tipo de cambio; la hipótesis esencial del NCM no es aceptable. Así, su política monetaria, que hace depender la inflación de la brecha de producto, constituye una contradicción para el modelo de crecimiento exportador de estos países y representa un método recesivo de estabilización de precios.
\end{abstract}

Palabras clave: inflación, política monetaria, tipo de cambio, reservas internacionales, banco central.

Clasificación JEL: C02, E31, E43, E52, E58, F43, P44, O54.

\section{The Modus Operandi of New Consensus Macroeconomics in Brazil, Chile and Mexico}

\begin{abstract}
This text provides a critical analysis of the experiences of Brazil, Chile and Mexico, countries that have adopted the inflationary targeting monetary policy espoused by New Consensus Macroeconomics ( $\mathrm{NCM}$ ). Using cointegration and error correction models, as well as a discussion of the major facts of the case, we contrast the NCM with the empirical evidence available. One essential feature of its modus operandi in these economies lies in the fact that inflation control is crucially dependent on the appreciation of the exchange rate. The essential hypothesis of the NCM cannot be accepted. Similarly, its monetary policy, in which inflation depends on the product gap, contradicts the export-led growth model adopted by these countries and is a recessive method of stabilizing prices.
\end{abstract}

Key Words: Inflation, monetary policy, exchange rate, international reserves, central bank.

* Facultad de Economía, UNAM, México. aidagarcia.lazaro@gmail.com, iph@unam.mx, respectivamente. 


\section{MODE OPÉRATOIRE DU NOUVEAU CONSENSUS MACROÉCONOMIQUE AU BRÉSIL, AU CHILI ET AU MEXIQUE \\ Résumé}

Les auteurs analysent avec un regard critique l'expérience que connaissent le Brésil, le Chili et le Mexique avec les objectifs d'inflation du « nouveau consensus macroéconomique " (NCM). Au moyen de modèles de co-intégration et de correction des erreurs et à travers la discussion des principaux faits stylisés, nous mettons en contraste le NCM et l'évidence empirique disponible. Une caractéristique essentielle de son mode opératoire dans ces économies réside dans le fait que le contrôle de l'inflation dépend crucialement de l'appréciation du taux de change ; l'hypothèse essentielle du NCM n'est pas acceptable. Ainsi, sa politique monétaire, qui fait dépendre l'inflation de l'écart de production mondial, constitue une contradiction pour le modèle de croissance exportatrice de ces pays et représente une méthode récessive de stabilisation des prix.

Mots clés: inflation, politique monétaire, taux de change, réserves internationales, banque centrale.

\section{MODUS OPERANDI DO NOVO CONSENSO MACROECONÔMICO NO BRASIL, CHILE E MÉXICO \\ Resumo}

Analisa-se criticamente a experiência do Brasil, Chile e México com a política monetária de metas de inflação do Novo Consenso Macroeconômico (NCM). Por meio de modelos de cointegração e correção de erros e a discussão dos principais fatos estilizados contrastamos o NCM com a evidencia empírica disponível. Uma característica essencial do seu modus operandi nestas economias consiste em que: o controle da inflação depende crucialmente da valorização cambial; e a hipótese do NCM não é aceitável. Assim, sua politica monetária, que faz depender a inflação da brecha do produto, constitui uma contradição para o modelo de crescimento exportador destes países e representa um método recessivo de estabilização dos preços.

Palavras-chave: inflação, política monetária, tipo de cambio, reservas internacionais, banco central.

\section{巴西、智利和、墨西哥的宏观经济学新共识}

摘要:

本文就巴西、智利和, 墨西哥三国的经历提供了一个重要分析, 这三个国家 都是采用了宏观经济学新共识所倡导的通货膨胀目标制的货币政策。我们 通过使用协整检验和误差纠正模型, 并讨论案例的主要事实, 比较了宏 观经济学新共识的经验事实。这三个经济体的一个重要特征在于控制通货 膨胀主要取决于汇率升值。宏观经济学新共识的假设是不能被接受的。同 样，它所倡仪的货币政策，即通货膨胀依赖产出缺口，是与这三个国采用 的出口导向增长模式相矛盾的, 而且也是一个稳定价格的具有负面影响的 办法。

关键词: 通货膨胀 货币政策汇率国际储备中央银行 
Macroeconomic model building since Lucas day has largely consisted of first accepting that a real system determines equilibrium and then inventing imperfections in the economic structure, money system, or financial markets so that non-neutrality results. Such a model is New Keynesian if the result is the existence of a number of equilibria that are not necessarily at full employment and if policy is effective. Such a model is New Classical if the result is that a unique real equilibrium exists and if policy is ineffective.

Hyman Minsky (1992-1993: 77).

\section{INTRODUCCIÓN}

En los años ochenta del siglo xx la economía convencional convergió en la tesis de que el Banco Central (BC) es el único responsable de la inflación (Hetzel, 2004). La estanflación de los años setenta había conducido a la adopción de metas de crecimiento de la oferta monetaria (Friedman, 1970), cuya efectividad depende crucialmente de la estabilidad de la función de demanda real de dinero y de que ésta exhiba baja elasticidad respecto de la tasa de interés. Sin embargo, debido a la innovación financiera, a partir de los ańos noventa los agregados monetarios M1 y M2 se tornaron altamente sensibles a la tasa de interés, su relación con la inflación se volvió muy inestable. En consecuencia, la política de metas monetarias resultó inútil para controlar la inflación (Cecchetti y Groshen, 2009: 115) y varios países de América Latina y Europa, entre otros, procedieron a fijar el tipo de cambio de sus monedas vis-à-vis otras monedas fuertes para anclar la inflación. En la era de la desregulación financiera, no obstante, las anclas nominales de tipo de cambio son inviables como marco de política monetaria.

Así, en los años noventa cristalizó un doble consenso, primero, en torno de los beneficios que una macroeconomía de baja inflación representa para el crecimiento económico, el empleo y la maximización del bienestar y, segundo, acerca de la tasa de interés como reguladora de los precios para alcanzar una meta de inflación baja y estable: "Un desarrollo particularmente importante en este sentido ha sido la adopción de un enfoque de 'objetivo de inflación' para elaborar la política monetaria por parte de varios bancos centrales del mundo en los años 1990" (Woodford, 2003: 3). Este es el Nuevo Consenso Macroeconómico (NCM) que sostiene que el modelo de metas de inflación y régimen de tipo de cambio flexible con la tasa de interés de corto plazo como 
instrumento es el marco de política monetaria óptimo. Varios bancos centrales en América Latina, Asia, Europa y Oceanía han adoptado el NCM (Bernanke et al., 1999; Arestis et al., 2005; Roger, 2009).

En las líneas que siguen se analiza la experiencia de Brasil, Chile y México con la política monetaria de objetivos de inflación (PMOI). Después de esta introducción, presentamos: el modelo del NCM y una sucinta revisión de cierta literatura relevante; una evaluación empírica del modus operandi del NCM en los tres países antes aludidos y las conclusiones que se derivan de este análisis.

\section{EL MODELO DE ECONOMÍA ABIERTA DEL NCM}

El modelo del NCM supone que hay una relación directa entre la demanda agregada y la tasa de interés; la primera influye en la inflación mediante la curva de Phillips con expectativas propuesta por la teoría nuevo keynesiana (Blanchard y Galí, 2005). La función de reacción del BC — la regla de Taylor — vincula los movimientos de la tasa de interés del BC con la evolución de la inflación, determinando así el producto real, el empleo y la tasa de inflación.

Knut Wicksell (1898) fue el pionero del marco teórico en el que la tasa de interés regula los precios y, por tanto, permite asegurar un nivel objetivo de precios. En el modelo de una economía de crédito puro de Wicksell, el BC debe seguir una regla muy simple para conseguir un nivel constante de precios que estabilice la inflación (Berg y Jonung, 1999): incrementar (disminuir) la tasa de descuento $\left(r_{t}\right)$ cuando los precios aumentan (disminuyen), de suerte que la diferencia entre $r_{t}$ y la tasa natural de interés $\left(r_{n}\right)$ es función de la discrepancia entre el nivel de precios observado $\left(\pi_{t}\right)$ y el nivel de precios objetivo $\left(\pi^{*}\right)$ :

$$
r_{t}=r_{n}+\phi\left(\pi_{t}-\pi^{*}\right)
$$

Woodford (2003: 49), autor del marco teórico más sofisticado del NCM, sostiene que Wicksell (1965 [1898], 2005[1907]) preludió la estrategia de PMOI, actualmente en boga. Sin embargo, es conveniente aclarar que la economía de crédito puro de Wicksell es una abstracción teórica sin pretensión de relevancia empírica, que en su teoría el sistema bancario es el detonante de los procesos acumulativos de inflación y deflación cuando no prevalece la "tasa de interés normal" o de equilibrio, mientras que Woodford (2003) presenta un modelo de política monetaria de una cashless economy (economía sin fricción monetaria), sin sistema bancario donde el $\mathrm{BC}$ establece objetivos de 
tasas de interés para alcanzar el objetivo de inflación apropiado. A diferencia de Wicksell, Woodford (2003) sugiere que su modelo es relevante no sólo para la teoría sino también para la práctica de la política monetaria, para establecer "un objetivo operacional de tasa de interés del banco central" (op. cit.: 37).

La versión contemporánea de la norma de Wicksell, según Woodford (2003), es la regla de Taylor (1993), que puede expresarse así:

$$
r_{t}=r_{t-1}+\Psi\left(y_{t}-y^{*}\right)+\phi\left(\pi_{t}-\pi^{*}\right)
$$

donde $y_{t}$ es el producto observado, $y^{*}$ el producto potencial. ${ }^{1}$ La diferencia fundamental entre la norma de Wicksell y la regla de Taylor consiste en que la primera implica un nivel de precios estacionario y la segunda uno de precios no estacionario. ${ }^{2}$

El modelo canónico de la regla de Taylor analiza el caso de una economía cerrada en la que el вС manipula la demanda agregada con el propósito de que la brecha de producto $\left(y_{t}-y^{*}\right)$ sea consistente con la inflación objetivo $\pi^{*}$. La estabilización de la inflación implica, simultáneamente, un nivel de producción de pleno empleo; esta "divina coincidencia" (Blanchard y Galí, 2005) da como resultado la estabilidad macroeconómica. De acuerdo con los principios del NCM, la política monetaria tiene un solo objetivo (la estabilidad de la inflación) y un solo instrumento (la tasa de interés); puesto que el régimen de tipo de cambio es flexible, la autoridad monetaria no tiene metas intermedias ni realiza intervenciones en los mercados cambiarios (Bernanke y Mishkin, 1997: 101; Svensson, 2001; Hüfner, 2004).

Ball (1997, 1999 y 2000) y Svensson (1998) desarrollaron el modelo del NCM para una economía pequeña abierta, incluyendo el tipo de cambio $(e)$ en el modelo de Taylor (1993). La regla de política monetaria óptima de esta índole la expresamos así:

$$
r=\left(\frac{\alpha}{w}\right) y+\left(\frac{b}{w}\right) \pi^{*}-\left[\frac{1-w}{w}\right] e
$$

Donde $\alpha$ y $b$ son parámetros y $w$ es un factor de ponderación. Por otra parte, bajo el influjo de Calvo y Reinhart (2002), surgieron estudios empíricos analizando el papel del tipo de cambio en la PMOI donde se muestra que varios bancos centrales (вСs) no permiten la libre flotación de sus monedas, el fenómeno denominado fear of floating. 
Uno de los objetivos principales de nuestro escrutinio es analizar el desempeño de la tasa de interés y del tipo de cambio en el marco de política monetaria del NCM en economías pequeńas abiertas (Ball, 1999). Consideramos un modelo de economía abierta compuesto por una curva Is (ecuación 4), una curva de Phillips (ecuación 5) y el tipo de cambio determinado por la paridad de tasas de interés (ecuación 6 ); $y$ denota el producto, $e$ el tipo de cambio, $\pi$ la tasa de inflación y $r$ la tasa de interés.

$$
\begin{aligned}
& y_{t}=-\beta r_{-1} \delta e_{-1}+\lambda y_{-1}+\epsilon \\
& \pi_{t}=\pi_{-1}+\alpha y_{-1}-\gamma\left(e_{-1}-e_{-2}\right)+\eta \\
& e=\theta r+v
\end{aligned}
$$

Expresamos la curva de Phillips que incluye la tasa de interés realizando un paso recursivo de la ecuación (6) y sustituyendo (6.1) en (5) para obtener (5.1):

$$
\begin{aligned}
& e_{-1}=\theta r_{-1}+v_{-1} \\
& \pi_{t}=\pi_{-1}+\alpha y_{-1}-\gamma\left(\left[\theta r_{-1}+v_{-1}\right]-e_{-2}\right)+\eta
\end{aligned}
$$

Después arribamos a la expresión (7), que es nuestra ecuación apropiada para la estimación econométrica, con el fin de obtener los valores de $\rho$ y $\gamma$, los cuales denotan el efecto de la tasa de interés y del tipo de cambio respectivamente en la inflación.

$$
\pi_{t}=\pi_{-1}+\alpha y_{-1}-\rho r_{-1}+\gamma e_{-2}+\zeta
$$

En el presente estudio analizamos las experiencias de Brasil, Chile y México, países que exhibieron altas tasas de inflación promedio durante el quinquenio previo a la adopción del NCM $(261.7,19.6$ y 22.8\%, respectivamente). Brasil adoptó una PMOI en 1999, con una meta de un rango de 6-10\%, mismo que ha disminuido a $2.5-6.5 \%$ en 2008 y a $4.5+/-2 \%$ en 2012 . Ferrari y Fabres (2009) afirman que, después de más de una década de PMOI, Brasil padece una tasa de inflación media muy elevada (7.1\% en 1999-2008), a pesar de que la tasa de interés real fue una de las más altas del mundo (9.9\%). Esto ha reducido el crecimiento económico y ha incrementado el valor de la deuda pública. 
Favero y Giavazzi (2004) consideran que las fluctuaciones del spread el emerging market bond index (ЕмBI) se transmiten al tipo de cambio vía los flujos de capital; al aumentar el riesgo de default, el tipo de cambio sufre una devaluación real y aumenta la razón deuda/pIB. Dado que la volatilidad del tipo de cambio altera las expectativas inflacionarias, las fluctuaciones en el spread del ЕмBI inducen adicionales incrementos en la tasa de interés Selic (el instrumento de la PMOI de Brasil). Este círculo vicioso conduce a escenarios de dominancia fiscal que impiden que el BC cumpla su objetivo de inflación. Chile adoptó la PMOI en septiembre de 1990. Céspedes y Soto (2004) identifican dos fases de la política de estabilización chilena: en la primera (1991-1999), la autoridad monetaria estableció objetivos de inflación, de déficit de cuenta corriente y un tipo de cambio administrado. Sólo durante la segunda fase (2000-presente) ha prevalecido un régimen de PMOI completo con la inflación como único objetivo de la política monetaria. El proceso de estabilización fue lento debido a que al inicio prevalecían problemas de credibilidad imperfecta (Schmidt-Hebbel y Tapia, 2002; Schmidt-Hebbel y Werner, 2002). Así, el BC de Chile procuró minimizar la pérdida de producto. Morandé (2002), a su vez, estudia una década de experiencia chilena con la PMOI; considera que la "inflación endémica de Chile por fin ha sido derrotada", pues su nivel promedio de "3.4\% en 1999-2000" se compara bien con el de los países industrializados y con el objetivo del вс; concluye que "el traspaso ha disminuido permanentemente" (op. cit:: 623). Y México empezó su transición hacia la PMOI después de la crisis financiera de 1994-1995 (Carstens y Werner, 1999). Inició en 2001 con un objetivo de mediano plazo de $3+/-1 \%$. Ramos y Torres (2005) sostienen que la política del вм ha sido consistente con los principios de la pMOI, lo que ha facilitado un proceso de estabilización "exitoso" gracias a que la disciplina fiscal ha permitido un equilibrio de baja inflación mediante una postura monetaria restrictiva. La estabilización mexicana prueba que la política monetaria puede anclar la inflación en una economía pequeña abierta con tipo de cambio flexible (Ramos y Torres, 2005: 3). Galindo y Ros (2008), y Ros (2013), por el contrario, sostienen que la PMOr del вм adolece de un sesgo hacia la sobrevaluación monetaria debido a una política cambiaria asimétrica que neutraliza las presiones hacia la devaluación y no neutraliza la apreciación monetaria. Por lo anterior, Ros (2013: 137-141) concluye que la PMOI ha contribuido al estancamiento productivo de la economía mexicana. Finalmente, Mántey (2009: 73) sostiene que, debido al elevado traspaso del tipo de cambio, el вм "no ha abandonado el ancla cambiaria", por ello la PMOI no se aplica consistentemente en México. 


\section{HECHOS ESTILIZADOS}

Analicemos ahora el desempeño del tipo de cambio nominal y real, la inflación, el nivel de reservas internacionales (RI), la base monetaria (H) y el crecimiento económico de Brasil, Chile y México.

Primero, la relación entre el tipo de cambio real y la inflación. La tendencia decreciente de la tasa de inflación de Brasil (a partir de 2002) y de México (después de la crisis de 1994-1995) ha venido acompañada de la apreciación persistente del real brasileño y del peso mexicano. Esta tendencia sólo se ha visto alterada por las crisis nacionales e internacionales (véanse las gráficas 1 y 2). En el caso de Chile la tendencia inflacionaria parece seguir una dinámica distinta, esto puede deberse a que el efecto del tipo de cambio sobre la inflación actúa con rezago o tiene una intensidad menor: se pueden destacar tres periodos en los cuales la disminución de la inflación ha estado acompañada de la apreciación de la moneda: de marzo 2003 a marzo 2004; finales de 2008-inicios de 2009 y durante 2012 (véase la gráfica 3).

Segundo, crecimiento significativo de las reservas internacionales. Un fenómeno notable del modus operandi de la PMOI es la trayectoria de $\mathrm{H}$ y la tendencia de RI. Recientemente se ha observado un desacoplamiento en sus ritmos de crecimiento. Mientras $\mathrm{H}$ muestra una trayectoria casi lineal, RI exhibe un crecimiento mucho mayor. Esto no obstante que desde la perspectiva de sus fuentes RI es un componente de $\mathrm{H}$, lo cual parece significar que hay un comportamiento peculiar del crédito interno. En las gráficas 4 y 5 se observa el comportamiento de RI y H de Brasil y México. En ambos el valor de RI ha superado al de H. En Brasil RI se venía incrementando con antelación, pero no fue sino a partir de 2005 cuando su tasa de acumulación aumentó exponencialmente. En México el Bм decidió acumular reservas después de la crisis de 1994-1995 mediante varios esquemas de intervención cambiaria instrumentados en 1996 y 1997. Asimismo, la crisis de 2008-2009 ha afectado temporalmente el comportamiento de RI, pues se ve un ligero proceso de desacumulación tanto en Brasil como en México, recuperándose su ritmo de crecimiento después de algunos meses. Los datos de Chile muestran dos fases distintas: de 2000 a 2007 el nivel de RI se mantuvo alrededor de 14 o 15000 millones de dólares, mientras que $\mathrm{H}$ creció a un ritmo relativamente mayor. A partir de 2008 el ritmo de acumulación de reservas se incrementó, desde entonces ambas variables crecen más o menos a la misma tasa (véase la gráfica 6). 
Grática 1. Brasil: inflación y tipo de cambio real, 2000(1), 2014(2)

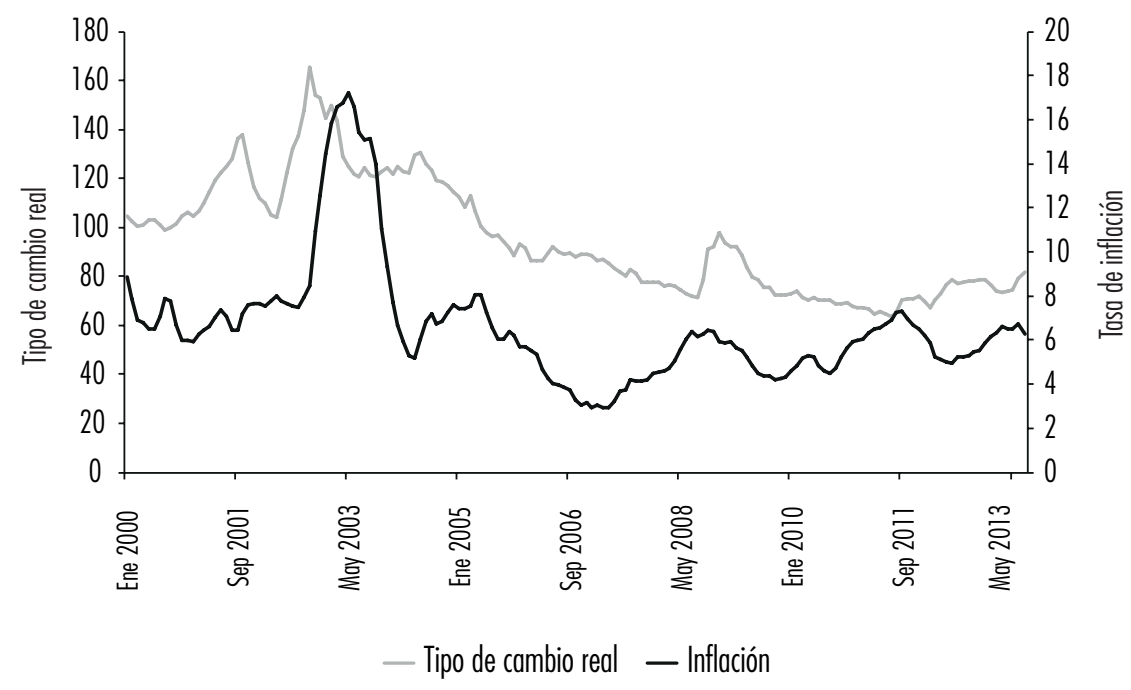

Fuente: elaboración propia con datos del IBGE y CEPAL.

Gráfica 2. México: inflación y tipo de cambio real, 1993(1)-2014(2)

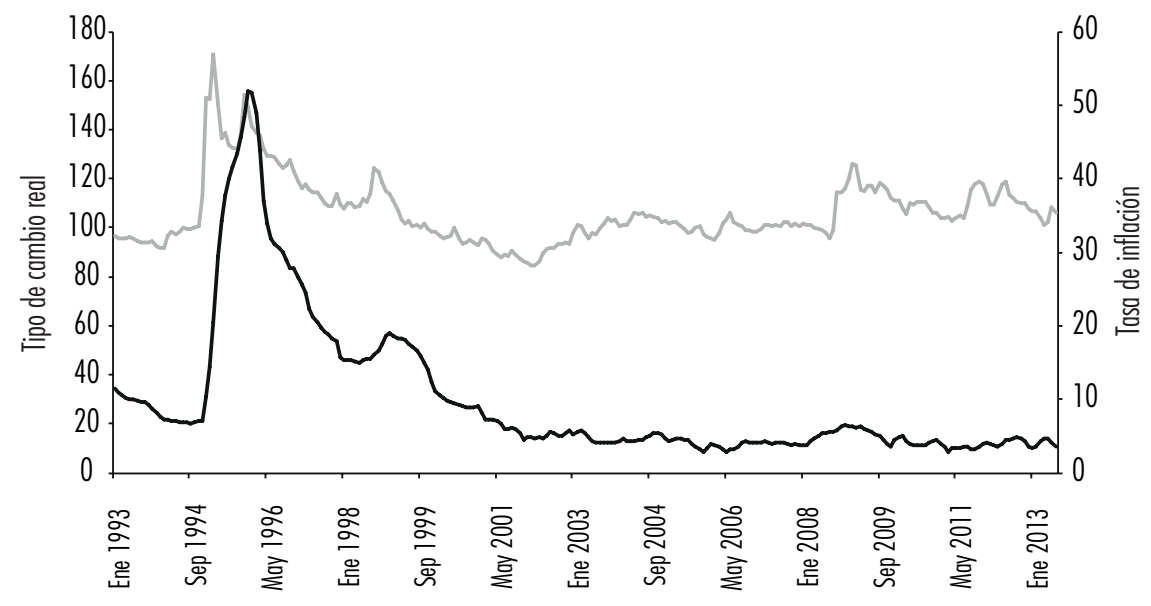

— Tipo de cambio real — Inflación

Fuente: elaboración propia con datos del INEG y Banco de México. 
Gráfica 3. Chile: inflación y tipo de cambio real, 2000(1)-2013(12)

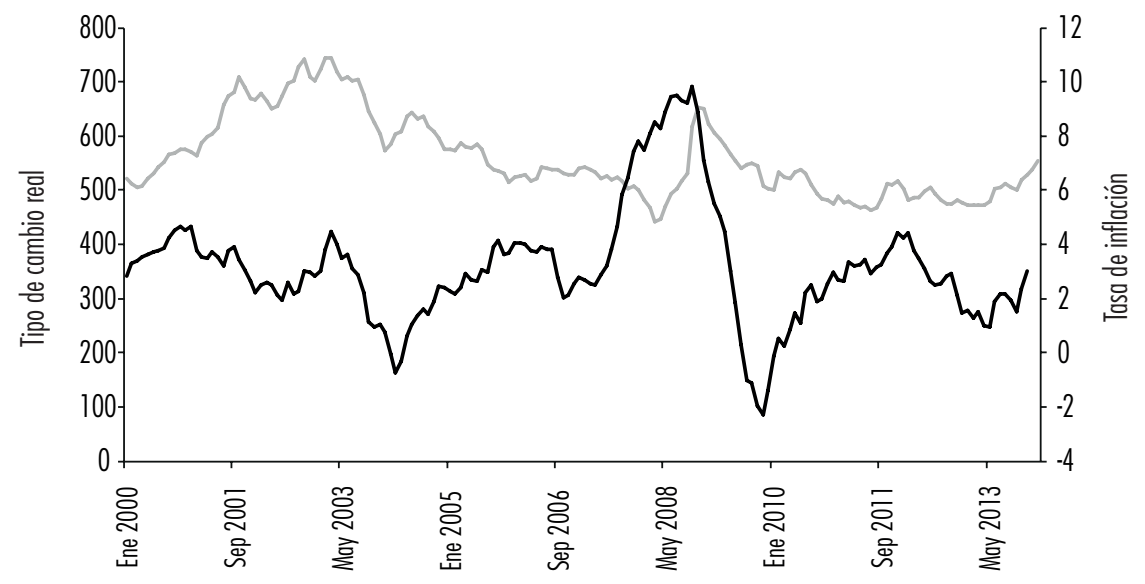

- Tipo de cambio real — Inflación

Fuente: elaboración propia con datos del INE y Banco Central de Chile.

Gráfica 4. Brasil: base monetaria y reservas internacionales, 2001 (1)-2013(10).

Índice base, diciembre de 2005

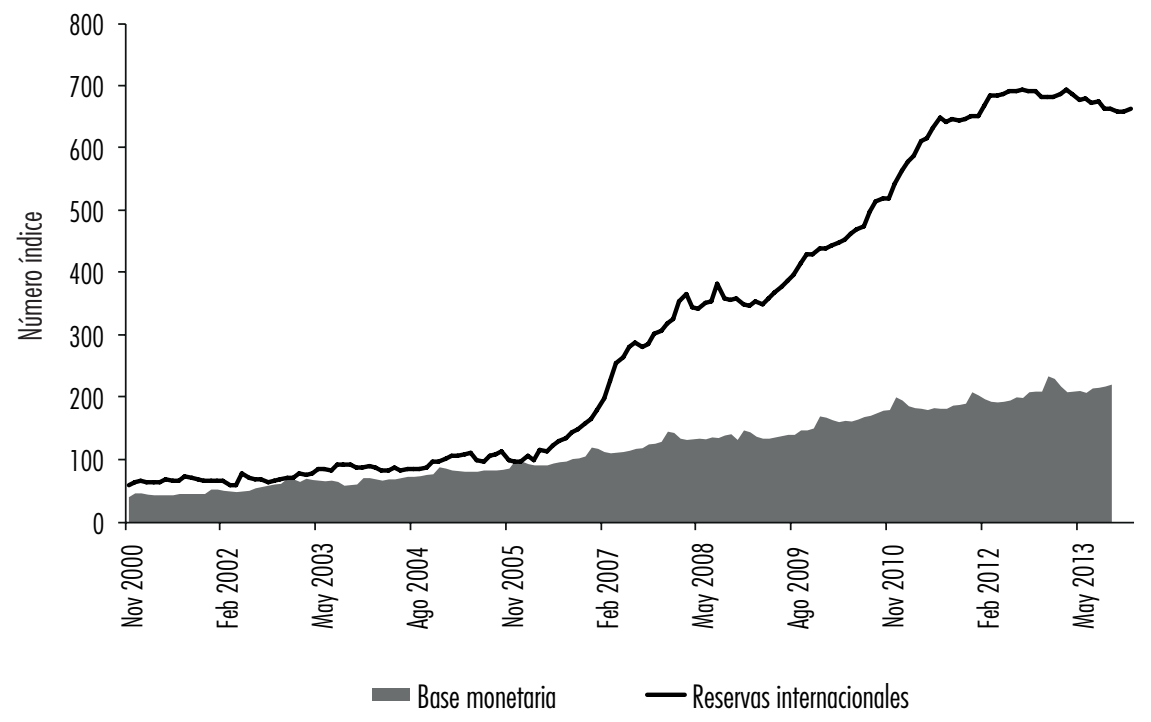

Fuente: elaboración propia con datos del Banco Central de Brasil. 
Gráfica 5. México: base monetaria y reservas internacionales, 1993(1)-2013(12).

Índice base, diciembre de 2005

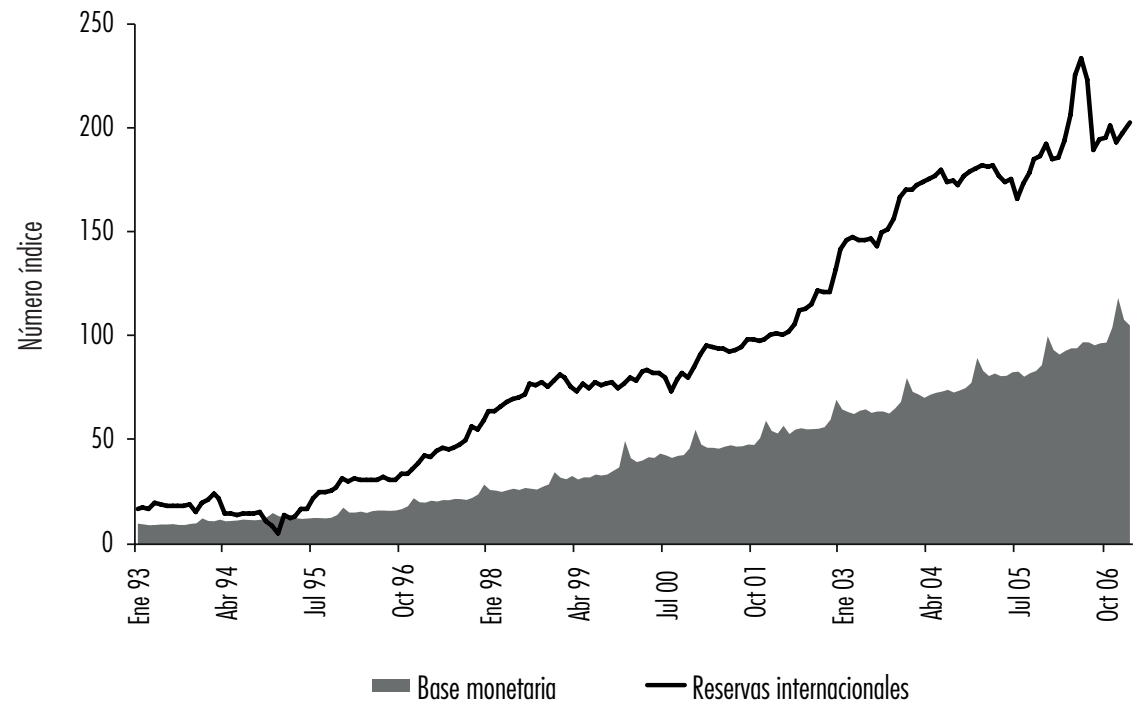

Fuente: elaboración propia con datos del Banco de México.

Gráfica 6. Chile: base monetaria y reservas internacionales, 2001(1)-2013(12).

Índice base, diciembre de 2005

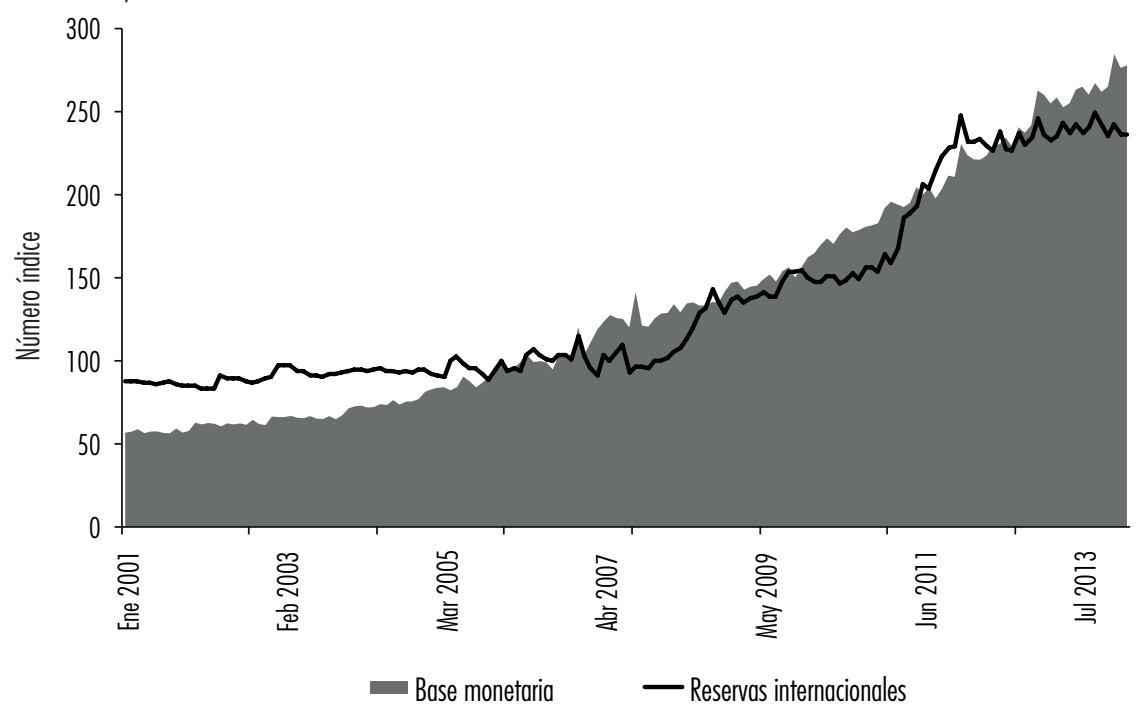

Fuente: elaboración propia con datos del Banco Central de Chile. 
Tercero, relación entre los tipos de cambio nominal y real y las reservas internacionales. Las monedas de las tres economías se han apreciado en paralelo a la disminución de la inflación. Esto se ha acompañado de un incremento significativo de RI (véanse las gráficas 7, 8 y 9): en Brasil han ocurrido sustanciales disminuciones del tipo de cambio que coinciden con periodos en que la acumulación de RI ha crecido (durante 2004, luego entre 2006 e inicios de 2008 y, finalmente, de 2009 a 2012). En México el peso se apreció en forma persistente de 1996 a 2003, proceso que se acompañó de dos esquemas de intervención que permitieron acumular rápidamente un monto de reservas que a juicio del Bм daba certidumbre a los mercados. En 2003 se anunció el cese de estos esquemas de intervención cambiaria y, entre otras razones, eso permitió el incremento de los tipos de cambio nominal y real. El segundo episodio de apreciación sustancial ocurrió a finales de 2008, cuando el BM realizó intervenciones extraordinarias que redujeron la volatilidad del tipo de cambio. A esto le siguieron varios esquemas de compra y venta de dólares, opciones y otros mecanismos de intervención cambiaria que dieron lugar a la acumulación significativa de RI y a la apreciación del peso. El caso de Chile tiene una dinámica algo distinta porque la relación entre la apreciación de la moneda y la acumulación de RI no es tan clara. En el largo plazo se registra la tendencia negativa del tipo de cambio, por un lado, y la acumulación excesiva de RI, por otro, particularmente después de 2007.

Cuarto, desaceleración del crecimiento económico. Al comparar el desempeño macroeconómico del periodo 1961-1980 (previo a la PMOI) con el de 1996-2013 (vigencia del PMOI), se constata una ralentización del crecimiento económico en la etapa más reciente. ${ }^{3}$ Brasil y México experimentaron tasas de crecimiento promedio anuales de 7.4 y $6.7 \%$ durante 1961-1980, con niveles de inflación de 43.4 y 11\%, respectivamente. En el periodo 1996-2013 Brasil y México crecieron 2.9 y $3.2 \%$, respectivamente; los niveles de inflación respectivos fueron 8.1 y $9.8 \%$. En Chile el crecimiento económico ha sido ligeramente mayor durante 1996-2013 en comparación con 1961-1980. Asimismo, la inflación ha descendido de manera significativa $(5$ y $116 \%$, respectivamente). La evidencia muestra que las tres economías han ganado en diferente grado en estabilidad de precios. Sin embargo, desde la perspectiva del crecimiento los efectos han sido adversos en Brasil y México. 
Gráfica 7. Brasil: tipos de cambio nominal y real y reservas internacionales, 2001 (1)-2014(2)

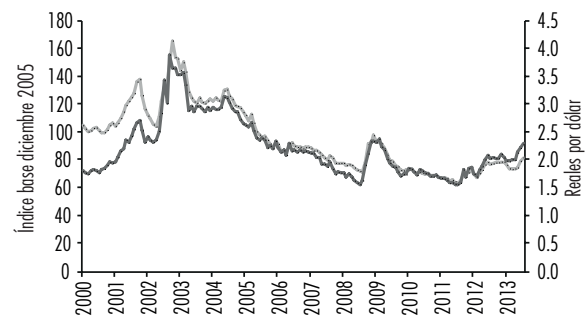

- Tipo de cambio real — - Tipo de cambio nominal

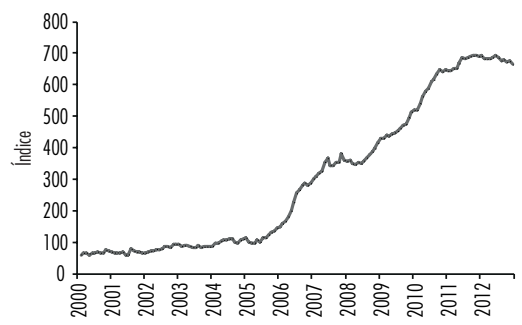

—Reservas internacionales

Fuente: elaboración propia con datos del Banco Central de Brasil y CEPAL.

Gráfica 8. México: tipos de cambio nominal y real y reservas internacionales, 1993(1)-2013(12)

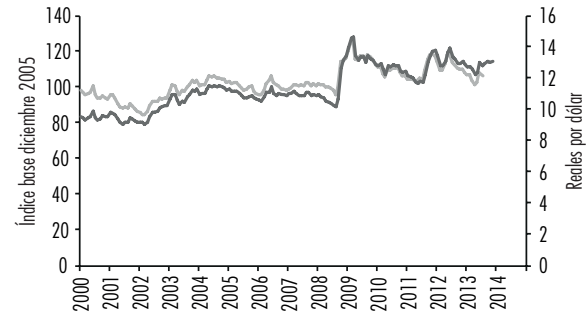

- Tipo de cambio real — -Tipo de cambio nominal

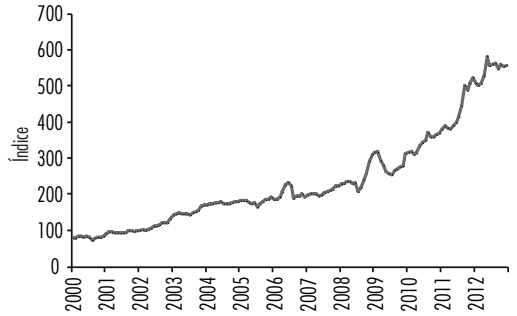

— Reservas internacionales

Fuente: elaboración propia con datos del Banco de México.

Gráfica 9. Chile: tipos de cambio nominal y real y reservas internacionales, 2001 (1)-2014(2)

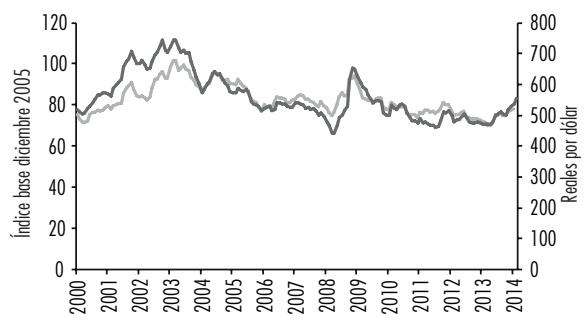

- Tipo de cambio real — -Tipo de cambio nominal

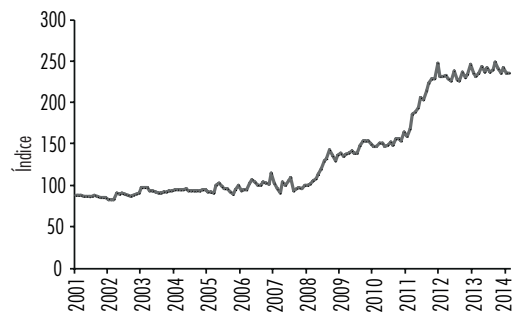

- Reservas internacionales

Fuente: elaboración propia con datos del Banco Central de Chile. 
Gráfica 10. Tasa de crecimiento anual del PIB en Brasil, México y Chile, 1961-1980 (\%)

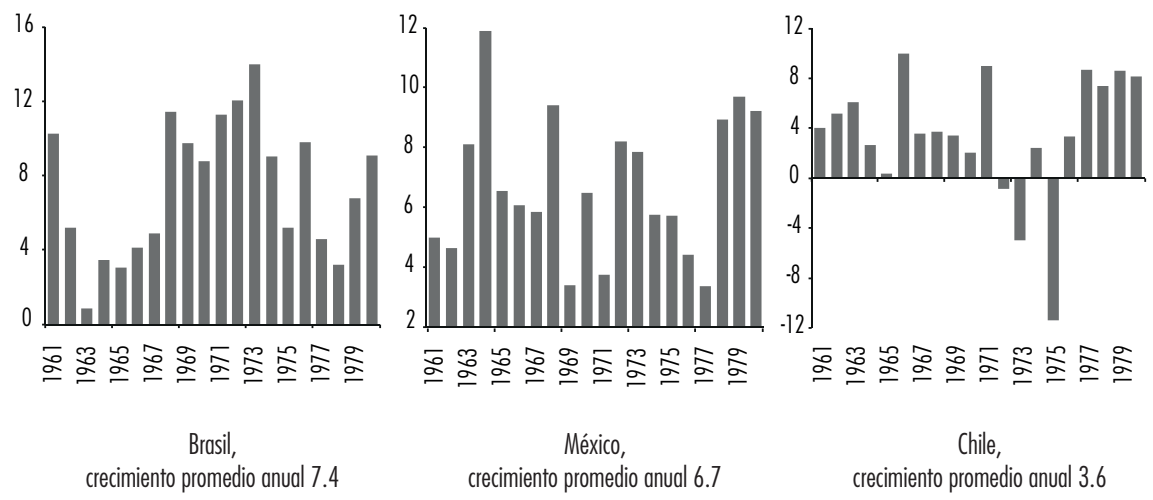

Fuente: elaboración propia con datos del Banco Mundial.

Gráfica 11. Inflación anual en Brasil, México y Chile, 1961-1980 (\%)

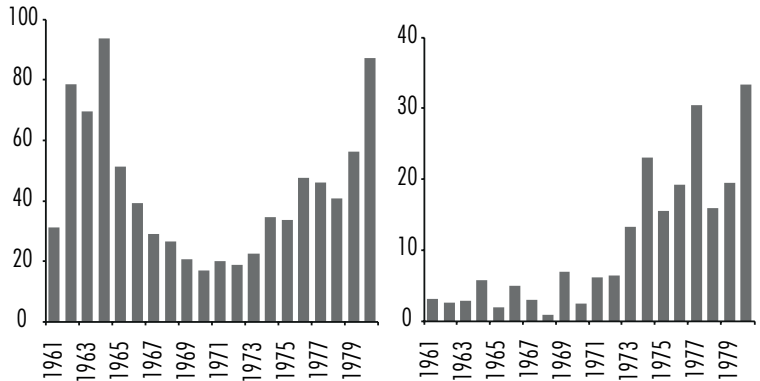

Brasil

1961-1971: inflación anual 43.5

1961-1980: inflación anual 43.4
México

1961-1971: inflación anual 3.9

1961-1980: inflación anual 11.0

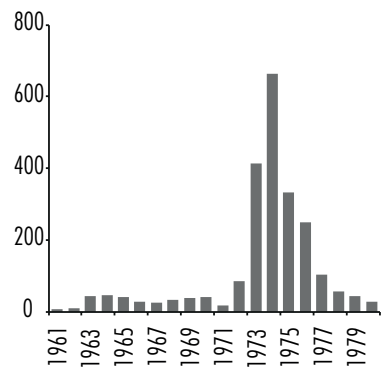

Chile

1961-1971: inflación anual 30.7

1961-1980: inflación anual 116.2

Fuente: elaboración propia con datos del Banco Mundial. 
Gráfica 12. Tasa de crecimiento anual del PIB en Brasil, México y Chile, 1996-2013 (\%)

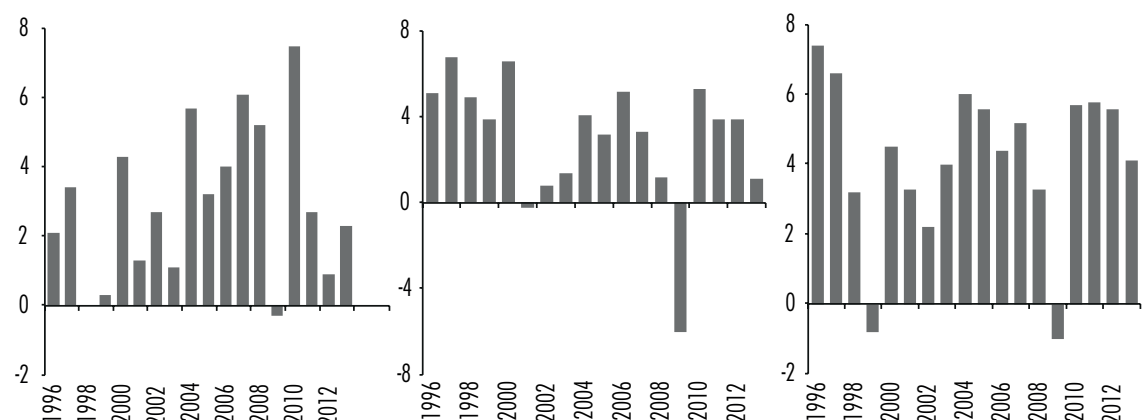

Brasil,

crecimiento promedio anual 2.9
México,

crecimiento promedio anual: 3.2
Chile,

crecimiento promedio anual 4.0

2004-2013 crecimiento promedio anual 3.7 por ciento.

Fuente: elaboración propia con datos del Banco Mundial.

Gráfica 13. Inflación anual en Brasil, México y Chile, 1996-2013 (\%)

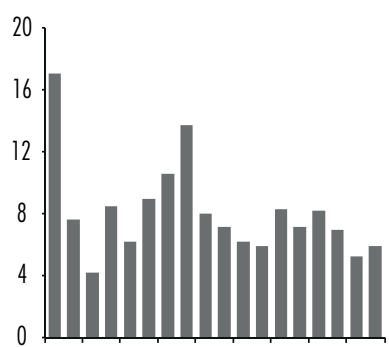

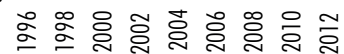

Brasil

1996-2013: inflación anual 8.1

2000-2013: inflación anual 7.9

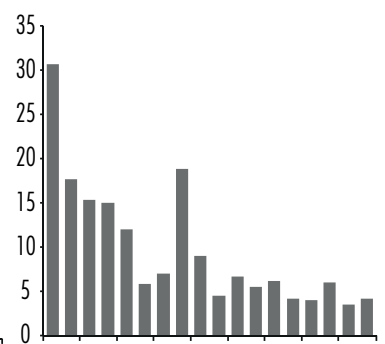

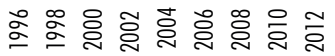

México

1996-2013: inflación anual 9.8

2000-2013: inflación anual 7.0

2004-2013: inflación anual 5.4

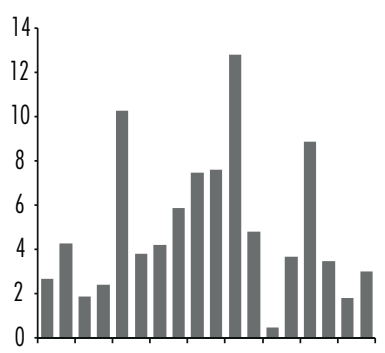

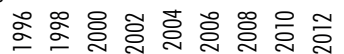

Chile

1996-2013: inflación anual 5.0 2000-2013: inflación anual 5.6

Fuente: elaboración propia con datos del Banco Mundial.

\section{ANÁLISIS EMPÍRICO}

Se analizaron las economías de Brasil, Chile y México durante el decenio 2003(1)-2013(12) en que ha estado vigente la PMOr. ${ }^{4}$ Se estimó la curva de Phillips para una economía abierta (ecuación 7); se supone que la tasa de 
inflación depende del producto $y$, la tasa de interés $r$ y el tipo de cambio con un periodo de rezago, así como de un efecto de ruido blanco $\zeta$. Además, se supone que $\alpha, \rho$ y $\gamma$ son parámetros positivos:

$$
\pi_{t}=\pi_{-1}+\alpha y_{-1}-\rho r_{-1}+\gamma e_{-2}+\zeta
$$

Se emplearon cuatro variables proxy, un índice de precios para aproximar la tasa de inflación, un indicador de la actividad económica para el producto, la tasa de interés de política monetaria y el tipo de cambio nominal. ${ }^{5}$ Se utilizaron las variables en logaritmos para estandarizar y reducir la dispersión. Las variables no son estacionarias, y su orden de integración es uno en todos los $\operatorname{casos}^{6}$ (véase el cuadro A.1 del anexo).

Se verificó la existencia de algún vector de cointegración; dado que las series no son estacionarias la estimación de la curva de Phillips por mínimos cuadrados ordinarios no es una opción econométrica apropiada. De acuerdo con las pruebas de la traza y máximo valor se encontró que hay al menos un vector de cointegración en cada uno de los países de la muestra (las pruebas se pueden ver en el cuadro A. 2 del anexo). Los vectores de cointegración se muestran en el cuadro 1; se confirma la presencia de una relación de largo plazo entre los precios, el tipo de cambio, la tasa de interés y el producto en las tres economías. Los coeficientes obtenidos son de diferente magnitud en cada economía, sin embargo guardan algunas semejanzas relevantes. Para las tres economías los precios responden con mayor elasticidad a los movimientos en el tipo de cambio que a los cambios en la tasa de interés, como se observa en las filas primera y tercera del cuadro 1. En Chile el efecto pass-through se ha reducido considerablemente, un incremento de $1 \%$ en el tipo de cambio genera un incremento de sólo $0.14 \%$ en la inflación. El efecto de la tasa de interés sobre los precios es marginal. Con relación a México, la depreciación del peso en $1 \%$ eleva la inflación en $0.40 \%$ y los incrementos de la tasa de interés en $1 \%$ reducen la inflación en sólo $0.16 \%$. Tanto en Chile como en México la elasticidad de los precios respecto al tipo de cambio es comparativamente mayor que la elasticidad de los precios vis-à-vis la tasa de interés. El caso de Brasil es un poco distinto en la medida que las elasticidades obtenidas para el tipo de cambio, la tasa de interés y el producto son mayores a las estimadas para las economías mexicana y chilena. Asimismo, la brecha entre el coeficiente estimado del tipo de cambio y la tasa de interés es menor que en los casos de México y Chile. La depreciación en 1\% de la moneda brasileña genera un incremento de la inflación en $0.5 \%$, mientras que un incremento en $1 \%$ en la 
Cuadro 1. Vectores de cointegración por Johansen para el periodo 2003(1)- 2013(12)

\begin{tabular}{lrrr}
\hline Modelo & México & \multicolumn{1}{c}{ Chile } & \multicolumn{1}{c}{ Brasil } \\
\hline$e_{\mathrm{f}}$ & 0.415 & 0.143 & 0.506 \\
s.e. & {$[-0.210]$} & {$[-0.139]$} & {$[-0.259]$} \\
$\mathrm{r}_{\mathrm{f}}$ & -0.159 & -0.027 & -0.25 \\
s.e. & {$[-0.070]$} & {$[-0.064]$} & {$[-0.140]$} \\
$y_{t}$ & 0.753 & 0.878 & 1.113 \\
s.e. & {$[-0.244]$} & {$[-0.197]$} & {$[-0.052]$} \\
Constante & 0.477 & & \\
& {$[-1.040]$} & & \\
\hline
\end{tabular}

Nota: Entre corchetes se anota el error estándar de los estimadores.

Fuente: elaboración propia con base en resultados econométricos.

tasa de interés reduce la inflación en $0.25 \%$. Esto sugiere que el вC de Brasil podría influir más en la inflación con sus instrumentos de política monetaria que sus homólogos mexicano y chileno; se puede presumir que el BC de Brasil tiene mayor margen de maniobra en la medida que la respuesta de la inflación a variaciones del tipo de cambio y la tasa de interés es mayor que en México y Chile.

De acuerdo con los resultados del modelo de largo plazo, el вм podría utilizar con mayor intensidad el tipo de cambio como instrumento de política, debido a su mayor efectividad para contener la inflación. En Chile ocurre lo mismo, si bien la efectividad es menor. Aunque no debe ignorarse que la apreciación del tipo de cambio deprime el crecimiento económico y el empleo.

El efecto del producto resultó positivo - como se esperaba- en todos los casos. La economía de Brasil exhibe la mayor elasticidad de los precios respecto al producto, seguida por la economía de Chile y, en tercer lugar, la economía mexicana. Esto muestra la elasticidad que tiene un вс para contener la inflación por la vía de la contracción económica, práctica utilizada con frecuencia. No obstante, debido al efecto de histéresis una política de enfriamiento persistente de la economía puede contraer la capacidad de expansión económica potencial (Perrotini, 2007; Ball, 2009).

Para capturar los efectos de corto plazo se estimaron modelos correctores de errores (MCE), tomando como base el vector de cointegración encontrado 
para cada país (véanse los cuadros 1 y 2). Respecto a los hallazgos de corto plazo, los modelos de las tres economías sugieren la convergencia al equilibrio como lo muestran los coeficientes de corrección que son significativos, menores a cero y mayores a menos uno. Los tres modelos pasaron las pruebas de especificación y estabilidad (véanse el cuadro A.3 y las gráficas A.1, A.2 y A.3).

Las estimaciones del cuadro 2 muestran que el nivel de precios se explica principalmente por sí mismo como un efecto autorregresivo. En consecuencia, resultaron significativos los coeficientes del primero y cuarto rezagos de los precios en la estimación de la economía mexicana, el primer rezago para la economía chilena y el primero, séptimo y noveno rezagos en el modelo para Brasil. Como se aprecia en los modelos, el desempeño actual de los precios está estrechamente relacionado con su pasado, principalmente el inmediato. El comportamiento de los precios en la economía brasileña guarda una memoria de más largo plazo. Además de la importancia de los precios del pasado para explicar la trayectoria de los precios actuales, resultaron estadísticamente significativos el tipo de cambio y la tasa de interés en el modelo chileno. Mientras que para las economías mexicana y brasileña los modelos reportaron significativos: el tipo de cambio, la tasa de interés y el producto. El coeficiente del tipo de cambio fue el segundo más elevado, sólo superado por los coeficientes de los precios rezagados. En el modelo de la economía mexicana es significativo el primer rezago del tipo de cambio, lo cual muestra la rapidez con la que actúa esta variable sobre la inflación. En la economía chilena el tercer rezago resultó significativo. En contraste, en el modelo de Brasil el séptimo es significativo, lo que sugiere que el tipo de cambio afecta a los precios con mayor lentitud. Así, la velocidad con que el tipo de cambio afecta a la inflación es muy alta en México y Chile y menor en Brasil.

En términos del efecto del tipo de cambio sobre los precios, en la economía mexicana la variación porcentual del tipo de cambio en $1 \%$ genera también una en los precios en $0.02 \%$ después del primer periodo del cambio en el valor de la moneda; en el largo plazo este efecto aumenta a $0.4 \%$ como se documenta por las relaciones de largo plazo. En el caso de Chile la variación del tipo de cambio en $1 \%$ genera una variación positiva de $0.03 \%$ en los precios después de tres periodos. El efecto de largo plazo alcanza $0.15 \%$ (véase el cuadro 1 ), valor marginal cuando se compara con los coeficientes de largo plazo de México y Brasil. El desempeño de la economía brasileña es interesante: a pesar de que el índice de precios se explica en buena medida por su pasado, el tipo de cambio tiene un efecto significativo. Sin embargo, esto ocurre con mayor lentitud en comparación con las economías mexicana y chilena. Asimismo, el coeficiente del tipo de cambio es el menor de los tres. Por otra parte, en los tres 
casos el coeficiente de la tasa de interés es menor que el estimado para el tipo de cambio; esta característica persiste en los modelos de corto y largo plazos. En la estimación para México la tasa de interés resultó significativa en el séptimo rezago, lo cual muestra que su velocidad en el efecto sobre los precios es mucho menor que con la que actúa el tipo de cambio: mientras éste afecta a los precios con un periodo de rezago, la tasa de interés lo hace con siete periodos de atraso. En la economía chilena la tasa de interés repercute en los precios con mayor prontitud: resultaron significativos el tercero y quinto rezagos; sin embargo, el efecto es transitorio pues el coeficiente del quinto rezago es de signo contrario y de igual magnitud que el valor del coeficiente obtenido en el tercer rezago. En las estimaciones de largo plazo la elasticidad de los precios, respecto a la tasa de interés, es marginal, de modo que los coeficientes de corto plazo confirman los resultados obtenidos arriba.

Cuadro 2. Modelo corrector de errores, 2003(1) - 2013(12)

\begin{tabular}{|c|c|c|c|c|c|}
\hline \multicolumn{2}{|c|}{ México } & \multicolumn{2}{|c|}{ Chile } & \multicolumn{2}{|c|}{ Brasil } \\
\hline Variable & Coeficiente & Variable & Coeficiente & Variable & Coeficiente \\
\hline \multirow[t]{2}{*}{$\Delta \mid p_{-1}$} & 0.417 & $\Delta p_{-1}$ & 0.384 & $\Delta l p_{-1}$ & 0.623 \\
\hline & {$[0.062]$} & & {$[0.077]$} & & {$[0.067]$} \\
\hline \multirow[t]{2}{*}{$\Delta \mid p_{-4}$} & -0.154 & $\Delta \mathrm{le}_{-3}$ & 0.039 & $\Delta \mid p_{-7}$ & -0.195 \\
\hline & {$[0.061]$} & & {$[0.0125]$} & & {$[0.063]$} \\
\hline \multirow[t]{2}{*}{$\Delta \mathrm{e}_{-1}$} & 0.029 & $\Delta \mathrm{lr}_{-3}$ & -0.008 & $\Delta p_{-9}$ & 0.268 \\
\hline & {$[0.009]$} & & {$[0.003]$} & & {$[0.062]$} \\
\hline \multirow[t]{2}{*}{$\Delta \mathrm{lr}_{-7}$} & -0.007 & $\Delta \mathrm{lr}_{-5}$ & 0.008 & $\Delta l e_{-7}$ & 0.009 \\
\hline & {$[0.003]$} & & {$[0.003]$} & & {$[0.005]$} \\
\hline \multirow[t]{2}{*}{$\Delta l_{-9}$} & 0.129 & $\delta_{0902}$ & -0.022 & $\Delta \mathrm{lr}_{-5}$ & -0.005 \\
\hline & {$[0.067]$} & & {$[0.004]$} & & {$[0.003]$} \\
\hline \multirow[t]{2}{*}{$\delta_{0605}$} & -0.008 & $\delta_{0812}$ & -0.015 & $\Delta \mathrm{y}_{-7}$ & 0.049 \\
\hline & {$[0.003]$} & & {$[0.003]$} & & {$[0.016]$} \\
\hline \multirow[t]{2}{*}{$\delta_{1001}$} & 0.008 & mce $_{-1}$ & -0.006 & $\delta_{0407}$ & 0.005 \\
\hline & {$[0.003]$} & & {$[0.001]$} & & {$[0.002]$} \\
\hline
\end{tabular}


Aída García Lázaro e Ignacio Perrotini

Continuación cuadro 2

\begin{tabular}{|c|c|c|c|c|c|}
\hline \multicolumn{2}{|c|}{ México } & \multicolumn{2}{|c|}{ Chile } & \multicolumn{2}{|c|}{ Brasil } \\
\hline Variable & Coeficiente & Variable & Coeficiente & Variable & Coeficiente \\
\hline \multirow[t]{2}{*}{$\delta_{1004}$} & -0.007 & & & $\mathrm{mce}_{.1}$ & -0.002 \\
\hline & {$[0.003]$} & & & & {$[0.001]$} \\
\hline \multirow[t]{2}{*}{$\delta_{1105}$} & -0.008 & & & & \\
\hline & {$[0.003]$} & & & & \\
\hline \multirow[t]{2}{*}{ mce $_{-1}$} & -0.013 & & & & \\
\hline & {$[0.002]$} & & & & \\
\hline
\end{tabular}

Fuente: elaboración propia con base en resultados econométricos.

En las estimaciones para Brasil el quinto rezago de la tasa de interés es significativo con un nivel de confianza de 90\%. Finalmente, el índice de actividad económica resultó significativo en México y Brasil y actúa con mayor lentitud que el resto de las variables, tal como se establece en los modelos teóricos comunes (Ball, 1997:4). Además de las variables relevantes, se incluyeron algunas variables dummies en los modelos que capturaron algún efecto particular en cada una de las economías, por ejemplo, la crisis internacional de 2008-2009.

\section{CONCLUSIONES}

En el análisis precedente del modus operandi del NCM en Brasil, Chile y México hemos examinado las hipótesis de la PMOI contrastándolas con los hechos estilizados y la evidencia empírica relevantes. El escrutinio de los datos estadísticos ponderados en nuestros modelos econométricos nos conduce a varias inferencias que cuestionan la supuesta superioridad del modelo de objetivos de inflación postulado por el Nuevo Consenso Macroeconómico.

Primero, en Brasil, Chile y México la inflación es más sensible a las fluctuaciones del tipo de cambio que a los movimientos de la tasa de interés: la elasticidad de largo plazo de los precios vis-à-vis el tipo de cambio es significativamente mayor que la relativa a la tasa de interés. Aunque hay diferencias de grado en cada caso, en general, la tasa de interés es insuficiente para controlar 
la inflación, razón por la cual los BC de estos países utilizan el tipo de cambio como un instrumento adicional efectivo para alcanzar el objetivo de la política monetaria: en ninguno de estos países los BC conducen su política antiinflacionaria con estricto apego al canon del NCM, sino sobre la base de un índice de condiciones monetarias con ponderaciones distintas para la tasa de interés y el tipo de cambio. Por tanto, se rechaza la hipótesis de estabilidad de precios postulada por el Nuevo Consenso Macroeconómico.

Segundo, los $\mathrm{BC}$ consiguen de facto su objetivo de inflación realizando intervenciones esterilizadas en los mercados cambiarios, evitando así que la volatilidad del tipo de cambio altere la meta de inflación baja y estable. Sin embargo, esto introduce un velo de opacidad en la política monetaria y viola el precepto de transparencia que, en teoría, caracteriza a la política monetaria de objetivos de inflación.

Tercero, la evidencia empírica muestra que los precios son elásticos respecto a la actividad económica en las tres economías (el coeficiente que arroja el modelo de largo plazo para Brasil es particularmente significativo). Esto implica que las contracciones del producto contribuyen a abatir la inflación. Este mecanismo recesivo para alcanzar el objetivo de inflación baja y estable ha sido empleado con cierta frecuencia. Amén de que el método recesivo es una derivada del modelo del NCM — la inflación depende de la brecha de producto-, tiene efectos de histéresis negativos de largo plazo sobre el crecimiento económico potencial.

Cuarto, analizando las características distintivas y específicas de cada uno de los países estudiados, observamos que en Brasil: a) la inflación pasada influye con más vigor en la presente, acusando así un efecto de inflación inercial; b) la elasticidad de los precios respecto a las variaciones de la actividad económica es mayor que en Chile y México, con lo que el potencial grado de conflicto entre inflación y crecimiento económico parece ser mayor ahí; c) la velocidad con que los ajustes en la tasa de interés afectan a la inflación es mayor que la de los movimientos del tipo de cambio, y d) dadas las elasticidades de la inflación vis-à-vis el tipo de cambio, la tasa de interés y el producto, el вс de Brasil puede influir en la inflación con mayor efectividad que los BC de Chile y México, pero con significativas pérdidas de producto y empleo.

En el caso de Chile, encontramos que: a) el tipo de cambio influye en la inflación con mayor fuerza que la tasa de interés, aunque el coeficiente de traspaso ha disminuido; b) los efectos de las fluctuaciones del tipo de cambio sobre los precios se transmiten más rápidamente que en la economía brasileña; c) el fenómeno de inflación inercial no es significativo, y d) la apreciación del tipo de cambio nominal tiene efectos nocivos en el producto potencial. 
En México: a) el tipo de cambio influye en la inflación con mayor vigor y velocidad que la tasa de interés; b) el coeficiente de traspaso ha disminuido, pero no al grado de anular el "miedo a flotar" el tipo de cambio; c) el efecto de la brecha de producto en la inflación es menor que en Brasil y Chile; d) al igual que los Bc de Brasil y Chile, el Banco de México parece tener incentivos para manejar el tipo de cambio de manera asimétrica (pasivamente ante apreciaciones monetarias y en forma activa para neutralizar presiones devaluatorias).

Quinto, la PMOI centrada exclusivamente en la estabilidad de precios no consiguió aislar a las economías de Brasil, Chile y México ante los choques de oferta ocurridos entre 2007 y 2009 (i.e., la volatilidad de los precios de las commodities - energéticos y alimentos - y la crisis financiera detonada por la bancarrota del mercado de hipotecas subprime y del banco Lehmann Brothers): estos choques de oferta no tuvieron relación alguna con la demanda agregada, sin embargo, causaron inestabilidad monetaria y real, y forzaron intervenciones de los $\mathrm{BC}$ y de los gobiernos con políticas fiscales "discrecionales". Los choques de oferta recientes expusieron de forma manifiesta que la tasa de interés no es un instrumento contracíclico eficiente, que el modus operandi del NCM no ha resuelto de raíz las tendencias procíclicas endémicas de la economía real y de los sistemas financieros de los países en cuestión: la fragilidad financiera y la restricción de balanza de pagos aún persisten, a pesar del contexto de inflación baja y estable.

El flagrante conflicto (trade-off) entre el modelo de inflación y el de crecimiento exportador vigentes en Brasil, Chile y México, ha derivado en la paradoja de un retorno a políticas activas de tipo de cambio en el seno del NCM cum régimen de tipo de cambio flexible. Esto significa que la causa causans de la inestabilidad macroeconómica reside en la estructura de la economía. En suma, hay bases para sostener que es imperativo que los BC de los países aquí analizados consideren objetivos de empleo y crecimiento económico en sus marcos de política monetaria, tal como lo hacen otros BC, por ejemplo, la Reserva Federal de Estados Unidos.

\section{NOTAS}

1 La regla de Taylor es formalmente similar a la norma de Wicksell si y sólo si $\Psi=0$.

2 Keynes (1923) aconsejó que la autoridad monetaria debía regular la oferta monetaria de suerte que "el número índice de los precios no se alejará de un punto fijo". Esta "norma" de Keynes es similar a la de Wicksell.

3 Las gráficas 10 y 12 muestran las tasas de crecimiento de 1961-1980 y 199 -2013 respectivamente, las gráficas 11 y 13 muestran las tasas de inflación de los mismos periodos. Se omite la 
década de los ochenta y los primeros años de la década de los noventa, debido a las crisis económicas que ocurrieron y a la década perdida.

4 Se han omitido los periodos de transición hacia la adopción de este marco de política, i.e., en el caso de Chile la fase de convergencia durante los años noventa, en el de Brasil el periodo de crisis de 2001 y 2002, y la fase de transición gradual en México (1995-2002).

5 La información de las variables relevantes, así como del indicador global de actividad económica (IGAE) para México, el indicador mensual de actividad económica (IMACEC) para Chile y el índice de actividad económica para Brasil, se tomó de los bancos centrales respectivos.

6 Para probar la no estacionariedad se realizaron tres pruebas de raíz unitaria: Dickey-Fuller Aumentada (ADF), Phillips-Perron (pP) y KPss (Dickey y Fuller, 1981; Phillips y Perron, 1988; Kwiatkowski et. al., 1992). Los resultados se muestran en el cuadro A.1 del anexo.

\section{ANEXOS}

Cuadro A1. Pruebas de raíz unitaria

\begin{tabular}{|c|c|c|c|c|c|c|c|c|}
\hline \multirow[t]{2}{*}{ Variable } & \multicolumn{3}{|c|}{$A D F$} & \multicolumn{3}{|c|}{$P P$} & \multicolumn{2}{|c|}{ KPSS } \\
\hline & A & B & C & A & B & $C$ & D1 & D2 \\
\hline \multicolumn{9}{|l|}{ México } \\
\hline$e_{t}$ & -3.515 & -1.976 & 0.673 & -3.013 & -1.699 & 0.822 & 0.070 & 1.169 \\
\hline$\Delta e_{t}$ & -8.974 & -9.001 & -8.981 & -8.886 & -8.914 & -8.96 & 0.040 & 0.036 \\
\hline$p_{t}$ & -2.188 & -1.045 & 3.671 & -3.333 & -0.909 & 9.801 & 0.117 & 1.496 \\
\hline$\Delta p_{t}$ & -4.053 & -4.15 & -1.981 & -7.931 & -7.708 & -5.156 & 0.027 & 0.074 \\
\hline$y_{t}$ & -3.1 & -0.313 & 2.592 & -2.221 & -0.179 & 2.838 & 0.107 & 1.396 \\
\hline$\Delta y_{t}$ & -4.563 & -4.589 & -2.941 & -3.471 & -3.656 & -3.114 & 0.071 & 0.078 \\
\hline$r_{t}$ & -3.075 & -2.61 & -2.26 & -3.154 & -2.628 & -1.888 & 0.113 & 1.045 \\
\hline$\Delta r_{t}$ & -10.399 & -10.377 & -10.214 & -10.498 & -10.559 & -10.441 & 0.090 & 0.125 \\
\hline \multicolumn{9}{|l|}{ Chile } \\
\hline$e_{t}$ & -3.458 & -3.227 & -0.822 & -2.673 & -2.583 & -0.879 & 0.155 & 0.947 \\
\hline$\Delta e_{t}$ & -7.83 & -7.724 & -7.698 & -7.621 & -7.634 & -7.632 & 0.053 & 0.144 \\
\hline$p_{t}$ & -1.592 & -0.455 & 4.333 & -1.767 & -0.425 & 4.576 & 0.161 & 1.387 \\
\hline$\Delta p_{t}$ & -8.657 & -8.69 & -4.735 & -8.881 & -8.912 & -7.656 & 0.100 & 1.387 \\
\hline$y_{t}$ & -2.471 & -0.827 & 4.728 & -2.245 & -0.795 & 4.884 & 0.157 & 1.352 \\
\hline$\Delta y_{t}$ & -14.025 & -14.06 & -12.227 & -14.059 & -14.093 & -12.412 & 0.060 & 0.081 \\
\hline$r_{t}$ & -3.088 & -3.085 & -1.451 & -2.167 & -2.139 & -0.988 & 0.096 & 0.105 \\
\hline$\Delta r_{t}$ & -3.847 & -3.865 & -3.878 & -3.991 & -4.009 & -4.021 & 0.053 & 0.054 \\
\hline
\end{tabular}


Aída García Lázaro e Ignacio Perrotini

Continuación cuadro Al

\begin{tabular}{|c|c|c|c|c|c|c|c|c|}
\hline \multirow[t]{2}{*}{ Variable } & \multicolumn{3}{|c|}{$A D F$} & \multicolumn{3}{|c|}{$P P$} & \multicolumn{2}{|c|}{ KPSS } \\
\hline & A & B & $c$ & A & $B$ & $c$ & D1 & $\mathrm{D} 2$ \\
\hline \multicolumn{9}{|l|}{ Brasil } \\
\hline$e_{t}$ & -1.722 & -2.609 & -1.044 & -1.244 & -2.148 & -1.066 & 0.266 & 0.894 \\
\hline$\Delta e_{t}$ & -8.848 & -8.552 & -8.543 & -8.775 & -8.577 & -8.578 & 0.037 & 0.48 \\
\hline$p_{t}$ & -2.43 & 0.131 & 5.515 & -2.892 & -0.945 & 13.838 & 0.156 & 1.41 \\
\hline$\Delta p_{t}$ & -6.522 & -6.609 & -3.305 & -6.422 & -6.522 & -3.221 & 0.142 & 0.168 \\
\hline$y_{t}$ & -2.272 & -0.956 & 3.291 & -2.298 & -0.956 & 3.279 & 0.171 & 1.383 \\
\hline$\Delta y_{t}$ & -11.512 & -11.533 & -10.699 & -11.511 & -11.533 & -10.795 & 0.04 & 0.074 \\
\hline$r_{t}$ & -2.631 & -2.628 & -1.919 & -3.258 & -2.564 & -1.545 & 0.072 & 1.18 \\
\hline$\Delta r_{t}$ & -11.05 & -10.917 & -10.77 & -11.393 & -11.281 & -11.199 & 0.045 & 0.149 \\
\hline
\end{tabular}

Nota: Se probaron tres modelos en los test Dickey Fuller Aumentada (ADF) y Phillips-Perron (PP) y dos modelos en la prueba KPSS. Los modelos de las dos primeras pruebas se definieron como sigue: modelo A incluye tendencia y constante, modelo B incluye constante y modelo C sin constante ni tendencia. Sus valores críticos respectivos son -3.44, -2.88 y -1.94. En el caso de la prueba KPSS el modelo D1 incluye tendencia y constante, mientras que el modelo D2 sólo incluyó intercepto. Los valores críticos respectivos son 0.146 y 0.463 . En todos los casos los valores críticos asumen un nivel de confianza de $95 \%$. Se pusieron negritas cuando las series fueron estacionarias de acuerdo con cada prueba y modelo.

Fuente: elaboración propia con base en resultados econométricos.

Cuadro A2. Pruebas de la traza y máximo valor

\begin{tabular}{lccccccc}
\hline $\begin{array}{l}\text { Hipótesis Ho: } \\
\text { No VC }\end{array}$ & eigenvalor & Traza & Traza crítica & Prob* & $\begin{array}{c}\text { Máximo } \\
\text { eigenvalor }\end{array}$ & $\begin{array}{c}\text { Valor crítico } \\
\text { al 95\% }\end{array}$ & Prob* $^{*}$ \\
\hline México & & & & & & & \\
\hline Ninguno & 0.244 & 77.344 & 54.079 & 0.000 & 42.826 & 28.588 & 0.000 \\
Máximo 1 & 0.110 & 34.518 & 35.193 & 0.059 & 16.415 & 22.300 & 0.159 \\
Máximo 2 & 0.086 & 18.103 & 20.262 & 0.097 & 13.777 & 15.892 & 0.28 \\
\hline Chile & & & & & & & \\
\hline Ninguno & 0.270 & 63.468 & 40.175 & 0.000 & 39.972 & 24.159 & 0.000 \\
Máximo 1 & 0.120 & 23.496 & 24.276 & 0.063 & 16.269 & 17.797 & 0.084 \\
Máximo 2 & 0.054 & 7.227 & 12.321 & 0.303 & 7.086 & 11.225 & 0.242 \\
\hline Brasil & & & & & & & \\
\hline
\end{tabular}




\begin{tabular}{lccccccc}
\hline $\begin{array}{l}\text { Hipótesis Ho: } \\
\text { No VC }\end{array}$ & eigenvalor & Traza & Traza crítica & Prob* & $\begin{array}{c}\text { Máximo } \\
\text { eigenvalor }\end{array}$ & $\begin{array}{c}\text { Valor crítico } \\
\text { al } 95 \%\end{array}$ & Prob* $^{\star}$ \\
\hline Ninguno & 0.267 & 55.286 & 24.159 & 0.000 & 38.836 & 24.159 & 0.000 \\
Máximo 1 & 0.094 & 16.450 & 17.797 & 0.311 & 12.345 & 17.797 & 0.393 \\
Máximo 2 & 0.032 & 4.105 & 11.225 & 0.427 & 4.080 & 11.225 & 0.479 \\
\hline
\end{tabular}

* Se utilizaron p-values de MacKinnon-Haug-Michelis (1999).

Fuente: elaboración propia con base en resultados econométricos.

Cuadro A3. Pruebas de correcta especificación

\begin{tabular}{lcll}
\hline Modelo & México & Chile & Brasil \\
\hline Jarque-Bera & 1.36 & 4.24 & 0.32 \\
Prob & 0.50 & 0.12 & 0.85 \\
LM Test & $10.50^{\star}$ & $5.43^{\star \star}$ & $3.15^{\star}$ \\
Prob & 0.31 & 0.37 & 0.96 \\
ARCH Test & $10.37^{\star}$ & $2.42^{\star \star}$ & $7.86^{\star}$ \\
Prob & 0.32 & 0.79 & 0.55 \\
Breusch-Pagan-Godfrey & 8.81 & 8.32 & 3.58 \\
Prob & 0.55 & 0.30 & 0.89 \\
\hline
\end{tabular}

*Ji-cuadrada 9 g.l.; ;*Ji-cuadrada 5 g.l.

Fuente: elaboración propia con base en resultados econométricos.

Gráfica A1. Pruebas de estabilidad del modelo corrector de errores de México
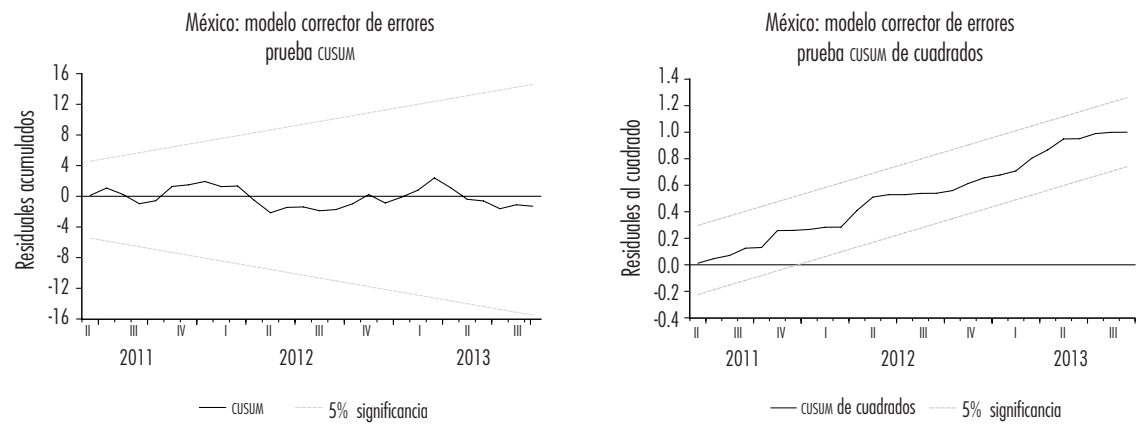

Fuente: elaboración propia con base en resultados econométricos. 
Gráfica A2. Pruebas de estabilidad del modelo corrector de errores de Chile
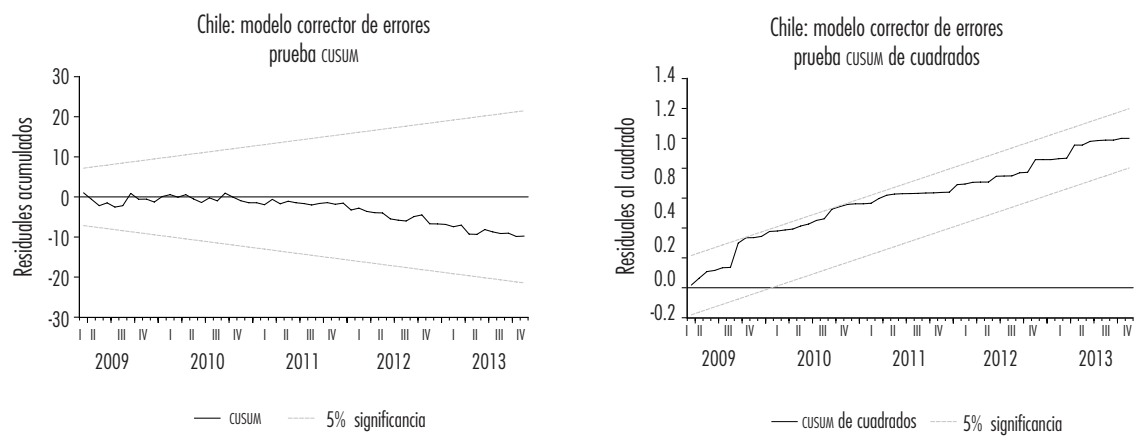

Fuente: elaboración propia con base en resultados econométricos.

Gráfica A3. Pruebas de estabilidad del modelo corrector de errores de Brasil
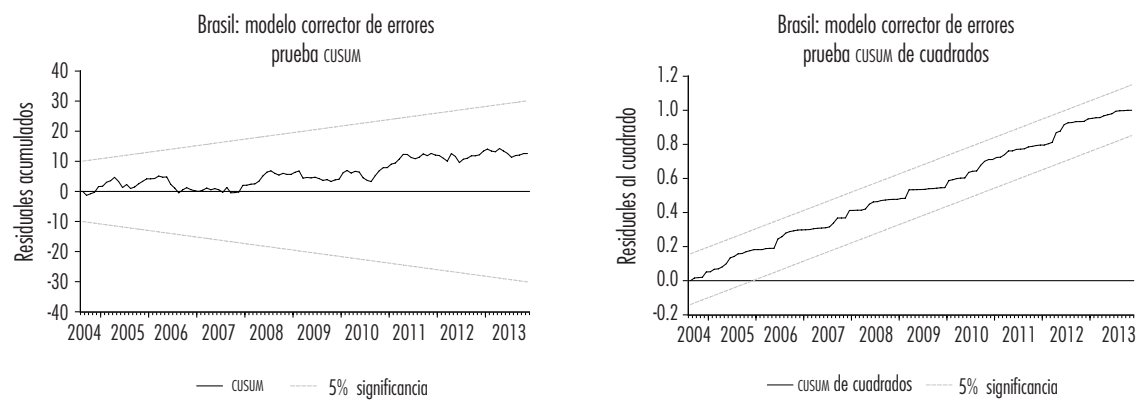

Fuente: elaboración propia con base en resultados econométricos.

\section{BIBLIOGRAFÍA}

Arestis, Phillip; Michelle Baddeley, y John McCombie (2005), The new monetary policy: Implications and relevance, Cheltenham, R.U., Edward Elgar, pp. 264.

Ball, Laurence (2009), "Hysteresis in unemployment: Old and new evidence”, NBER Working Papers, núm. 14 818, Massachusetts, NBER, marzo, pp. $1-37$.

(2000), "Policy rules and external shocks", Central Bank of Chile Working Papers, núm. 82, Santiago de Chile, Banco Central de Chile, octubre, pp. 1-25.

(1999), "Policy rules for open economies", NBER Working Papers, núm. 6 760, NBER, octubre, pp. 1-30. 
Ball, Laurence (1997), "Efficient rules for monetary policy", NBER Working Papers, núm. 5 952, Massachusetts, NBER, marzo, pp. 1-24.

Bernanke, Ben S., y Frederic Mishkin (1997), "Inflation targeting: A new framework for monetary policy?", Journal of Economic Perspectives, vol. 11, núm. 2, Broadway, American Economic Association, pp. 97-116.

Bernanke, Ben S.; Thomas Laubach; Frederic S. Mishkin, y Adam S. Posen (1999), Inflation targeting: Lessons from the international experience, Princeton, Princeton University Press, pp. 392.

Berg, Claes, y Lars, Jonung (1999), "Pioneering price level targeting: The swedish experience 1931-1937, Journal of Monetary Economics, núm. 43, Estados Unidos, Elsevier, junio, pp. 525-551.

Blanchard, Olivier, y Jordi Galí (2005), "Real wage rigidities and the new keynesian model”, NBER Working Papers, núm. 11 806, Massachusetts, NBER, noviembre, pp. 1-36.

Calvo, Guillermo A., y Carmen M. Reinhart (2000), "Fear of floating”, NBER Working Papers, núm. 7 993, Massachusetts, NBER, noviembre, pp. 1-64.

Carstens, Agustín, y Alejandro Werner (1999), "Mexico's monetary policy framework under a floating exchange rate regime", Documentos de Investigación del Banco de México, núm. 9 905, México, Banco de México, mayo, pp. $1-52$.

Cecchetti, Stephen G., y Erica L. Groshen (2003), "Understanding inflation: implications for monetary policy”, en Jacques Drèze (ed.), Advances in macroeconomic theory, Nueva York, Palgrave Macmillan, pp. 113-135.

Céspedes, Luis F., y Claudio Soto (2005), "Credibility and inflation targeting in an emerging market: The case of Chile", Central Bank of Chile Working Papers, núm. 312, Santiago de Chile, Banco Central de Chile, abril, pp. $1-40$.

Dickey David, y Wayne Fuller (1979), "Distribution of the estimator for autorregresive time series with a unit root", Journal of American Statistical Association, vol. 74, Broadway, American Statistical Association, pp. 427431.

(1981), "Likelihood ratio statistics for autorregresive time series with a unit root", Econometrica, vol. 49, núm. 4, Estados Unidos, John Wiley \& Sons, Inc., pp. 1 057-1 072.

Favero, Carlo A., y Francesco Giavazzi (2004), "Inflation targeting and debt: Lessons from Brazil”, NBER Working Papers, núm. 10 390, Massachusetts, NBER, marzo, pp. 1-21.

Ferrari Filho, Fernando, y María Juliana Fabris (2009), "El régimen de metas de inflación en Brasil, 1999-2008 evaluación crítica y desempeño macro- 
económico", Investigación Económica, vol. LXviII, núm. especial, 2009, México, Facultad de Economía-unam, pp.147-167.

Friedman, Milton (1970), "A theoretical framework for monetary analysis", Journal of Political Economy, vol. 78, Chicago, University of Chicago Press, marzo-abril, pp. 193-238.

Galí, Jordi, y Mark Gertler (1999), "Inflation dynamics: a structural econometric analysis", Journal of Monetary Economics, vol. 44, núm. 2, Estados Unidos, Elsevier, octubre, pp. 195-222.

Galindo, Luis Miguel, y Jaime Ros (2008), "Alternatives to inflation targeting in México", International Review of Applied Economics, vol. 22, núm. 2, Estados Unidos, Routledge Taylor y Francis Group, pp. 201-214.

Hetzel, Robert L. (2004), "How do central banks control inflation?", Federal Reserve Bank of Richmond Economic Quarterly Review, vol. 90, núm. 3, Richmond, Federal Reserve Bank of Richmond, marzo, pp. 47-63.

Hüfner, Felix (2004), Foreign exchange intervention as a monetary policy instrument: Evidence for inflation targeting countries, Alemania, Physica Verlag.

Keynes, John M. [1923] (1991), "Notes for the lecture to the National Liberal Club", reimpreso en Collected Writings of John M. Keynes, vol. xIx, Londres, Macmillan.

Kwiatkowski, Denis; Peter Phillips; Peter Schmidt, y Yongcheol Shin (1992), Testing the null hypothesis of stationary against the alternative of a unit root", Journal of Econometrics, vol 54, North-Holland, Elsevier Science Publishers, pp. 159-178.

Mántey, Guadalupe (2009), "Intervención esterilizada en el mercado de cambios en un régimen de metas de inflación: la experiencia de México", Investigación Económica, vol. LXvIII, núm. especial de 2009, Facultad de Economía-UnAM, pp. 47-78.

(2013), “¿Conviene flexibilizar el tipo de cambio para mejorar la competitividad?”, Revista Problemas del Desarrollo, vol. 44, núm. 175, México, UNAM, octubre-diciembre, pp. 9-32.

Minsky, Hyman (1992-1993), "On the non-neutrality of money", FRBNY Quarterly Review, pp. 77-82.

Morandé, Felipe G. (2002), "A decade of inflation targeting in Chile: Developments, lessons, and challenges", en Norman Loayza, y Raimundo Soto (eds.), Inflation targeting: Design, performance, challenges, Santiago, Banco Central de Chile, pp. 583-626.

Perrotini, Ignacio (2007), "El nuevo paradigma monetario", EconomíaUNAM, núm. 11, México, FE-UnAM, mayo-agosto, pp. 64-82. 
Phillips, Peter C.B. (1987), "Time series regression with a unit root", Econometrica, vol. 55, núm. 2, Estados Unidos, John Wiley \& Sons Inc., pp. 277-302.

Phillips, Peter, y Pierre Perron (1988), "Testing for a unit root in time series regression”, Biometrika, vol. 75, núm. 2, Oxford University Press, pp. 335436.

Ramos Francia, Manuel, y Alberto Torres García (2006), "Inflation dynamics in México: A characterization using the new Phillips curve", Documentos de Investigación del Banco de México, núm. 2006.15., México, Banco de México, diciembre, pp. 1-24.

(2005), "Reducing inflation through inflation targeting: The mexican experience", Documentos de Investigación del Banco de México, núm. 2005.01., México, Banco de México, julio, pp. 1-26.

Roger, Scott (2009), "Inflation targeting at 20: Achievements and challenges”, IMF Working Papers, núm. 236, Nueva York, International Monetary Fund, octubre, pp. 1-32.

Ros, Jaime (2013), Algunas tesis equivocadas sobre el estancamiento económico de México, México, El Colegio de México-unam, pp. 156.

Schmidt, Hebbel, y Claudio Tapia (2002), "Inflation targeting in Chile", North American Journal of Economics and Finance, núm. 13, Estados Unidos, Elsevier, pp. 125-146.

Svensson, Lars (1998), "Open-economy inflation targeting", NBER Working Papers, núm. 6 545, Massachusetts, Estados Unidos, NBER, mayo, pp. 1-53. (2001), "Independent review of the operation of monetary policy in New Zealand: Report to the Minister of Finance", Reportes del Tesoro de Nueva Zelanda, núm. 1, Nueva Zelanda, El Tesoro de Nueva Zelanda, pp. $1-79$.

Taylor, John (1993), "Discretion versus policy rules in practice", CarnegieRochester Conference Series on Public Policy, 39 (0), pp. 195-214.

Wicksell, Knut [1898] (1965), Interest and prices, Nueva York, Augustus M. Kelley, pp. 337.

[1907] (2005), "The influence of the rate of interest on prices", The Economic Journal, vol. 17, núm. 66, Estados Unidos, John Wiley \& Sons, Inc., junio, pp. 213-220.

Woodford, Michael (2003), Interest and prices: Foundations of a theory of monetary policy, Princeton, Princeton University Press, pp. 765. 
